\title{
Elliptical Distance Transforms and Applications
}

\author{
Hugues Talbot \\ A2SI-ESIEE / IGM \\ 2 boulevard Blaise-Pascal \\ 93192 Noisy-le-Grand, France \\ h.talbot@esiee.fr
}

\begin{abstract}
Discrete Euclidean distance transforms, both exact and approximate, have been studied for some time, in particular by the Discrete Geometry community.

In this paper we extend the notion of Euclidean distance transform (EDT) to elliptical distance transform (LDT). The LDT takes an additional two fixed parameters (eccentricity and orientation) in 2-D and an additional four in 3-D (two ratios and two angles) in 3-D, instead of 1 for the EDT in all cases. We study first how the LDT can be computed efficiently with good approximation in the case where all parameters are constant.

We provide an application to binary object segmentation as motivation for this work.
\end{abstract}

\section{Introduction}

The discrete, non-Euclidean distance transform (DT) (e.g. underlying the 4-, 6or 8-connected grid) has been under study for quite some time, see for example [1], and more recently in arbitrary dimensions [2. Various efficient algorithms for computing the DT, linear in the number of pixels $N$, have been known since about the same time [3. This efficiency has led the DT to be used in a number of situations, for example to compute the skeleton [4, among others 5].

\subsection{Euclidean Distance Transform}

If one defines the DT of a discrete binary set as the function which associate each pixel to its shortest distance to the exterior of the set, one can also define a discrete Euclidean Distance Transform (EDT), simply substituting the Euclidean distance for the grid-based one. The EDT has several advantages, mainly isotropy, but is more costly to compute. There exists an obvious but unusable (quadratic in $N$ ) algorithm for computing this transform. Therefore practitioners have sought various reasonable and efficient approximation to the EDT [6]. In particular Danielsson [7] proposed a linear algorithm with excellent approximation.

Exact, linear algorithms for the EDT have recently been proposed in the literature, for example in 6-connectivity via chain propagation [8], by ordered propagation [9], using Voronoïdiagrams [10, and using dilation by paraboloid [1], among others. 


\subsection{Elliptical Distance Transform}

The Elliptical Distance Transform (LDT ${ }^{1}$ ) was defined in 12 can be understood as an extension of the Euclidean Distance Transform (EDT). We substitute the Euclidean distance ED between two points $p$ and $q$ (in 2-D)

$$
\operatorname{ED}(p, q)=\sqrt{\left(x_{p}-x_{q}\right)^{2}+\left(y_{p}-y_{q}\right)^{2}}
$$

by a new function $\mathrm{LD}$, with $\rho=\mathrm{ED}(p, q), \rho \cos (\theta)=x_{p}-x_{q}, \rho \sin (\theta)=y_{p}-y_{q}$, $\alpha$ an arbitrary angle and $\sigma=a / b$ with $a$ and $b$ pair of arbitrary real numbers:

$$
\mathrm{LD}(p, q)=\rho \sqrt{\frac{(b \cos (\theta-\alpha))^{2}+(a \sin (\theta-\alpha))^{2}}{\sigma}} .
$$

We have two extra parameters, it is easy to see that $\sigma=a / b$, is the axis ratio of an ellipse and $\alpha$ its orientation. The function LD has all the attributes of a distance (positivity, symmetry, $L D(a, b)=0 \Leftrightarrow a=b$, triangular inequality), and its level lines are all ellipses of orientation $\alpha$ and axis ratio $\sigma$. By convention $\sigma>0$, as the converse is equivalent to the convention with an additional rotation of $\pi / 2$ in $\alpha$. In the remainder of the paper, we assume that parameters $\alpha$ and $\sigma$ are arbitrary, but fixed for a given computation.

\subsection{Grey-Weighted and Geodesic Distance Transform}

In the usual DT, whether Euclidean or not, space is considered of constant metric, i.e. the transform is invariant by translation. An extension to usual DT is to consider the grey-weighted distance transform (GWTD) [13], for which the cost of going through a pixel is equal to the grey-level value of this pixel. This concept is clear in the discrete case as it reduces to computing min-cost paths on graphs 14. However, this idea also extends to the Euclidean DT, by considering a discrete image as as sampled continuous space with scalar metric [15]. In this case one is then numerically solving the Eikonal equation $|\nabla u|=g$, where $g$ is the sampled metric.

The classical way to solve this equation, and thus obtain a grey-weighted Euclidean distance transform is a discrete algorithm called the Fast Marching Method (FMM) [16. We note that by setting $g \equiv 1$ everywhere, with proper initial conditions, we compute the EDT - with two caveat. First the FMM is not linear in complexity, but only $O(N \log N)$ in the worst case (in practice very close to linear), with $N$ the number of pixels. However with arbitrary real values for $g$, and not simply limited discrete integer values this is in fact optimal, as a sorting pass is inevitable. Second the FMM is not exact but only first or second-order accurate.

Recently this work was extended to Riemannian metrics - i.e metrics that are positive definite on arbitrary manifolds, with the Ordered Upwind Method

\footnotetext{
${ }^{1}$ Given that ' $\mathrm{L}$ ' is pronounced as "ell" this acronym sounds adequate even if
} unorthodox. 
(OUM) [17. In other words, this allowed to compute the equivalent of a discrete positive definite tensor-weighted LDT. Perhaps more clearly, in DG terms this is a WDT for which at each point the weight is defined by a ratio and an orientation just like the LDT, and this weighting can vary from pixel to pixel. For a constant tensor, this obviously reduces to the LDT, with the same caveat as for the EDT vs. the FMM.

In the literature one finds mentions of the geodesic DT, which is a DT computed inside a binary set [18], for which a Euclidean version exists [19], however this reduces to the GWDT or FMM with infinite cost outside the set and constant positive cost within.

\subsection{Rest of the Paper}

In the following we propose an efficient algorithm for computing the LDT. This algorithm is accurate to within a fraction of a pixel.

\section{Computing the LDT}

The computation of the LDT following [20] is relatively simple as it is a straightforward adaptation of a known ordered propagation algorithm [21. This algorithm carries all the approximations of the Danielsson approach, but this is not usually a problem in most applications. More importantly, this adaptation is only valid for $\sigma$ ratios close to 1 , which limits its usefulness.

\subsection{Motivating Example}

An important problem in image analysis is the separation of touching binary particles for counting purposes. A series of methods have been proposed in various cases, but a recurring theme is when the shapes of the touching particles are close to disks. This is useful when counting blood cells or fibres in cross sections, for example.

The classical approach to this problem with mathematical morphology is to compute the watershed line on the complement of the distance transform [22]. To deal with border noise, one can use suitable h-maxima on the distance transform as markers [23]. Other approaches involving the analysis of the DT along the skeleton [24, using the conditional bisector [25] and later the bisector function [26, 27]. The latter approach is able to separate even the most deeply fused particles [28].

However when the particles are no longer disks but ellipses this approach breaks down, yet separating elliptical fused particles is also a common problem. For example eukariot cell nuclei are often elliptical in shape. Cylindric fibres in cross-section are not necessarily perpendicular to the cutting plane, and therefore their shape can be elliptical as well. In [20] the authors proposed to use a grid search of LDTs to find the centre of fused ellipses accurately. This is illustrated on Fig. 1. 


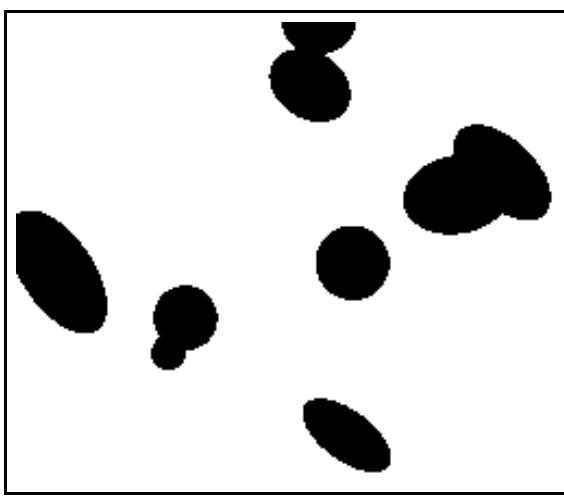

(a)

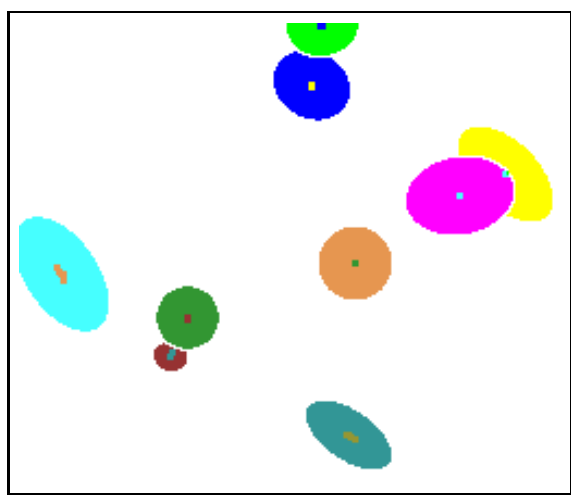

(c)

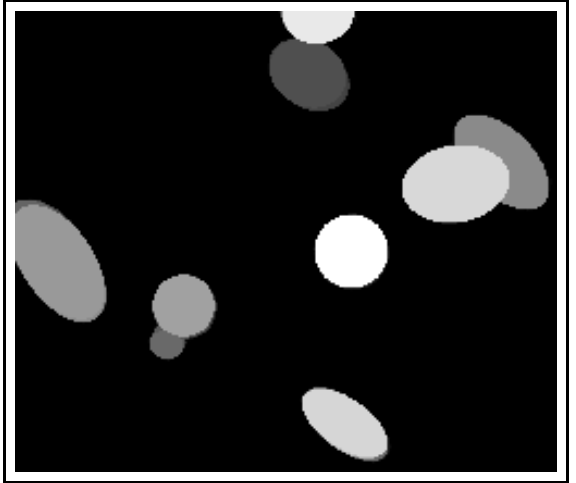

(b)

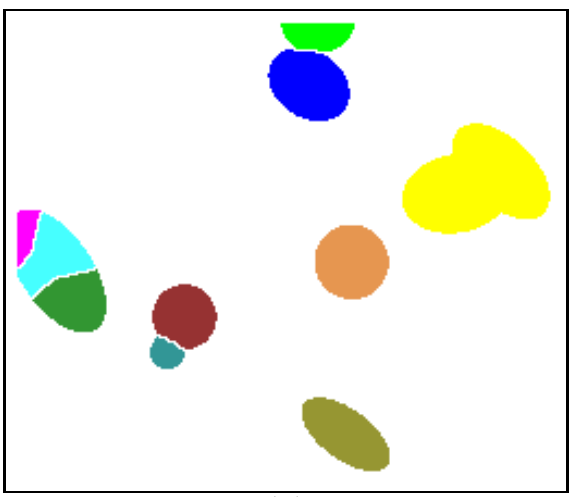

(d)

Fig. 1. Separation of fused ellipses: (a) binary input image ; (b) Fitted ellipses from the LDT search ; (c) segmentation result - each ellipse is drawn with a distinctive colour with their found centre. For comparison, (d) is the watershed segmentation based on the EDT

\subsection{Algorithm for Computing the LDT}

We only provide an explanation for the implementation in 2-D in the square grid, however the principle remains the same for higher dimensions and different grids. The basic idea is along the same vein as Dijkstra's algorithm and Fast Marching Methods in that each point is visited only once, and the neighbours of the point with the smallest distance are visited first.

Here the elliptical distance is defined as

$$
\sqrt{u^{2}+(\sigma * v)^{2}}
$$

where $u$ and $v$ are the distances along the principal axes of the ellipse and $\sigma$ is fixed.

Previous Algorithm. The specific algorithm is based on the algorithm described in 20]. In that algorithm, when each point is processed, one looks at its 
8-connected neighbours for which labelled point they are closest to. The algorithm uses this as the set of possible closest image points.

The distance to each of the possible labelled points is then calculated, and whichever is the closest of those is stored as the closest labelled point to the point being considered. The unlabelled points are put in a priority queue when one of their neighbours is processed, where the priority is the distance calculated for the point that added them.

The crucial assumption this algorithm makes however is that when a point is processed, its nearest labelled point is also the nearest labelled point of one of its already processed neighbours. For a discrete distance function such as the city-block and chessboard metrics it can be proven, with considerable work, that processing the points whose neighbours have the lowest distance first guarantees this to be the case. In the Euclidean case, most approximation methods make this assumption, which is false. However the error is in fact bounded and small.

However for an elliptical distance function this is not neccesarily the case. For high values of $\sigma$, the nearest labelled point will be one directly along the major axis (the $u$ axis) of the level lines of the distance, because even a small step along the minor axis (the $v$ axis) causes a large increase in the distance.

If the angle of the major axis is reasonably close to the angle of the edge of the object in the labelled image, the nearest point will therefore be quite a way along this edge, and therefore not one of the neighbours of the point being processed.

Neighborhoods. To solve this problem, each point no longer looks at just its 8-connected neighbours for possible nearest labelled points. Instead it considers its "neighbours" to be all points within a certain distance from itself ("distance" again being elliptical). Note that the points added to the queue for processing are still the 8-connected neighbours of the newly processed point, it is only when processing the points that the definition of 'neighbours' changes.

Clearly if this distance was big enough to include the entire image, then one of these "neighbours" always would have the same (or more specifically would be) the closest labelled point, however the algorithm would then become quadratic in complexity, and this is clearly not desirable. The question of what distance is the minimum to guarantee the accuracy of the algorithm (as the smaller the distance the faster it runs) has not yet been conclusively solved.

The value used in this function is just a little bit more than $\sigma$, designed to be approximately the smallest distance that still makes sure the 8 connected neighbours are a subset of the neighbour set. Also it should be noted that while the choice of defining the neighbours by the elliptical distance function being used has not been mathematically proven to be the best, it does intuitively seem a reasonable choice and, more importantly, gives good results.

Elliptical Distance. Another problem which had to be overcome was the fact that the distance between grid points could become significant for high values of $\sigma$. This is because a half pixel error in the distance along the minor axis is magnified by a factor of $\sigma$ and for pixels close to the labelled pixels can easily 
become bigger than the distance itself. To fix this, the distance between two grid points is re-defined as being the minimum distance between the first grid point and a 1 pixel wide square (i.e. half a pixel to each side).

To find the elliptical distance in terms of the known $x$ and $y$ co-ordinates, $u$ and $v$ are found in terms of $x$ and $y$ and substituted into the elliptical distance equation 3 . As the $u$ and $v$ axes are just a rotation by the angle $\alpha$ in Eq. 2 .

$$
\begin{aligned}
& u=x \cos (\alpha)+y \sin (\alpha), \\
& v=y \cos (\alpha)-x \sin (\alpha) .
\end{aligned}
$$

We actually compute the square of the LDT, as this allows all calculations and bookkeeping to be done in integer arithmetic. The square of the elliptical distance is then

$$
u^{2}+(\sigma v)^{2}=a x^{2}+b x y+c y^{2},
$$

where $a, b$ and $c$ depend only on $\sigma$ and $\alpha$.

To find the re-defined distance between two grid points, the distance to the closest point on the surrounding square of the second grid point needs to be found. The closest point must lie on either the horizontal side of the square visible to the the grid point or the vertical side visible (if neither horizontal side is visible the left is arbitrarily chosen to be looked at and similarly the bottom if neither vertical side is visible). If the closest point is not one of the corners it must be a local minimum of the distance function along that line, and more importantly, because there is only one local minimum along any line it must be the global minimum. For a horizontal line $y$ is fixed, and the (squared) distance function is just a quadratic in $x: a x^{2}+(b y) x+c y^{2}$. Elementary theory on quadratics gives the global minimum at $x=-b y /(2 a)$, and the minimum value as $y^{2}\left(c-\left(b^{2}\right) /(4 a)\right)$

Similarly for vertical lines the minimum is at $y=-b x /(2 c)$ and has value $x^{2}\left(a-\left(b^{2}\right) /(4 a)\right)$ The values $-b / 2 a$ and $-b / 2 c$ are precalculated and compared with $x / y$ or $y / x$ when needed. The distance is found by working out which two lines are visible, checking if the minimum of the distance function along those lines lies inside the square (if it does it is easy to show it will be the closest point on the square), and if it is not then calculating the distances to the three corners and taking the minimum.

\subsection{Pseudo-code}

In the pseudo code in Fig. 2, $P$ is identical to the LD function defined in Eq. 2,

\subsection{Results}

Accuracy. Fig 3 shows two level lines of the LDT of a set where the background constitutes a single point in the centre of the image. The dotted lines shows the LDT computed by the algorithm from 20, the solid line shows the proposed algorithm. The parameters were $\sigma=5$ and $\alpha=45^{\circ}$. 


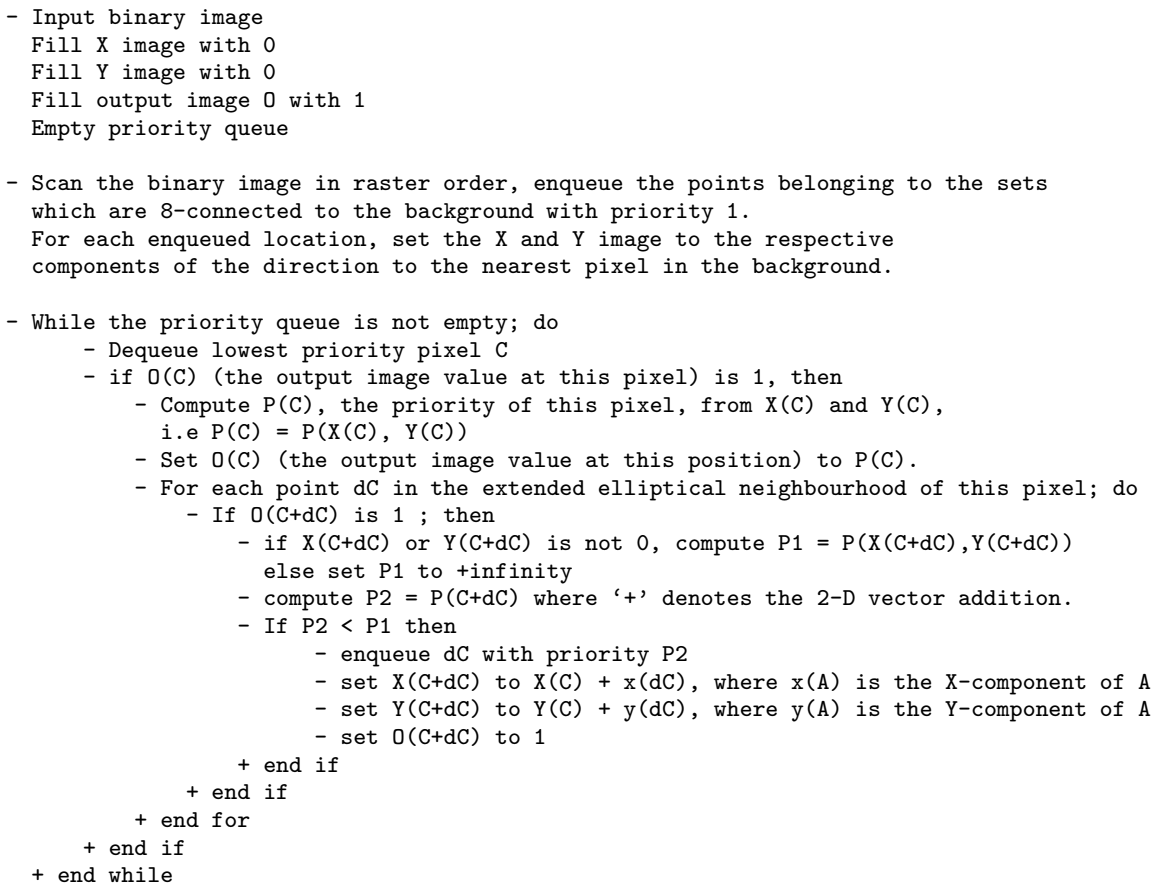

Fig. 2. Pseudo-code for the LDT. See text for the formulation of the priority function P.

The maximum distance between the largest level lines for both algorithms is approximately one pixel. The average distance is about 0.6 pixel. However, a comparison with the current algorithm and a parametric (drawn) ellipse with the same parameters shows no difference. On the negative side, the newer version of the algorithm takes approximately twice as long to compute, with these parameters, as it needs to inspect a larger neighbourhood during the propagation.

We did not include the result of a comparison with the OUM as it is quite inaccurate for this purpose. Being first-order accurate meaning that the error increase linearly with the distance. The OUM is also quite slow (approximately an order of magnitude) compared to these special-purpose LDT.

Application. Figure 5 shows a sample of an image of overlapping cell nuclei, together with the segmentation achieved using an ellipse fitting method using the current LDF algorithm described in 20] and reproduced in algorithm in Fig. 4:

Here, the space of possible $\alpha$ and $\sigma$ parameters is sampled in a grid search. More sophisticated optimisation methods can be envisaged, but this is not the purpose of this article.

For display purposes it is convenient to compute a measure which is high for a good fit. The measure that we use is $s=100 / \mathrm{m}$. We make sure that $s$ is never 0 even for a perfect fit. We call $s$ the score of a ellipse fit. To display the 


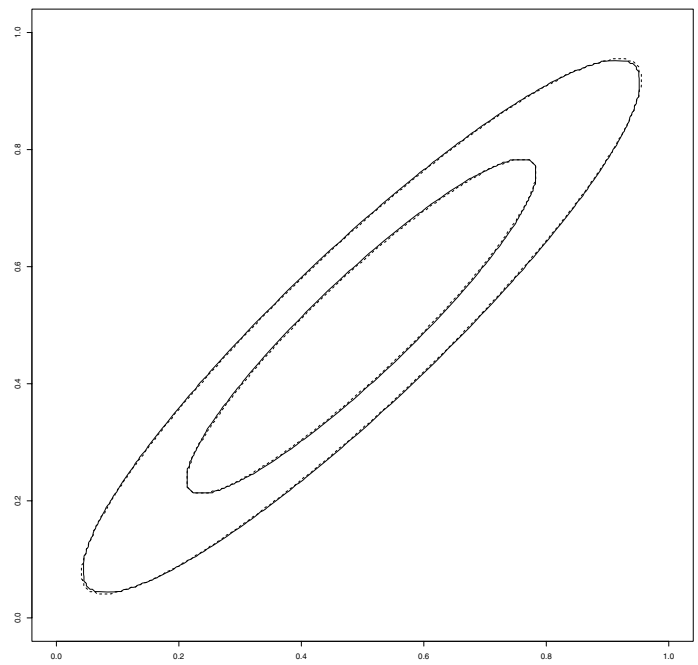

Fig. 3. Two level lines of the LDT computed by previous (dotted line) and current algorithm (solid line)

1. The EDM from the contour of the binary image is computed into image $C$.

2. A number of LDMs of the binary image are computed, with different values of $\alpha$ and $\sigma$.

3. For each LDM, special points are considered as candidate centres of ellipses. These need not include all the points of the LDM skeleton. In our application we only considered discrete regional maxima of the LDM.

4. For each special point, a candidate ellipse is generated with the same parameters (position, axis lengths and orientation) as the underlying LDM. Each pixel of the circumference of the generated ellipse is associated with its distance from the boundary of the binary image. This is provided by a simple interpolated lookup in image $C$. The pixel distances are sorted and a given percentile is taken as the measure $m$ of goodness-of-fit (for example $50 \%$ yields the median distance).

Fig. 4. Algorithm for ellipse fitting

result of the fit, we draw each candidate ellipse from lowest score to highest in the grey-level of their score. As an illustration, Fig 1)(b) shows the score of the fitted ellipse on the motivating example. We see that well-fitted ellipses tend to overwrite poorly-fitted ones.

On the real cell example, we sample the space with 31 different LDTs with $\delta \alpha=30^{\circ}$ and $\delta \sigma=0.5,1 \leq \sigma \leq 3$. The result of the ellipse fit appears reasonable, and table 1 confirms this impression. In this table we have in the first column the result of a careful manual count, in the second the result of the segmentation achieved by the proposed algorithm, the result of the segmentation achieved by the previous algorithm of 20, the result of the segmentation by watershed on an EDT, both unfiltered and filtered by h-maxima.

We see that on this sample the error rate with the new method is slightly improved compared with the older method, and much improved compared to the watershed method - it is 3 times lower. 


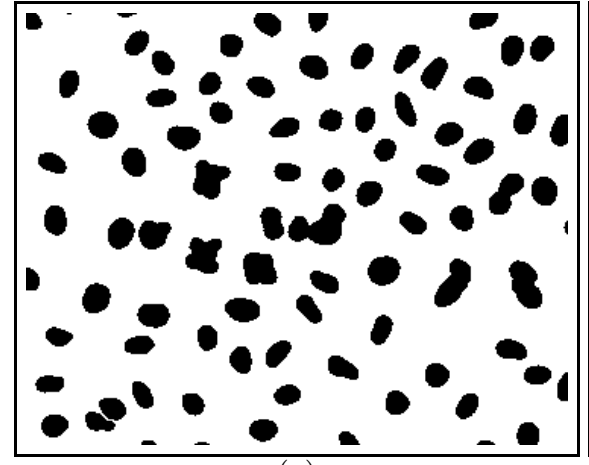

(a)

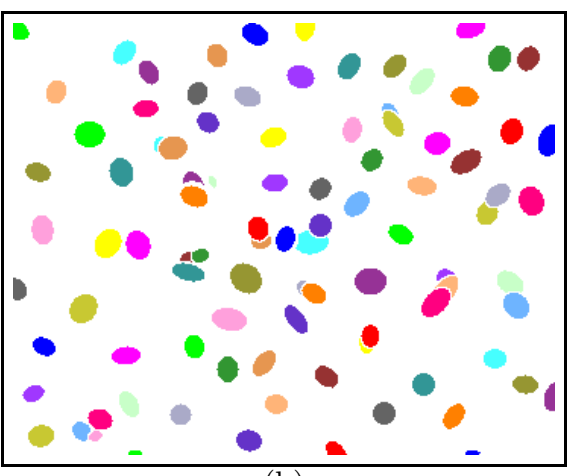

(b)

Fig. 5. Result of the algorithm on real data. (a) original image ; (b) segmentation using the overlapping ellipses method.

Table 1. Comparison of counting methods for image in Fig. 5(a)

\begin{tabular}{|l|l|l|l|l|l|}
\hline Count method & Manual & $\begin{array}{l}\text { Ellipse } \\
\text { high acc. }\end{array}$ & $\begin{array}{l}\text { Ellipse } \\
\text { Low acc. }\end{array}$ & $\begin{array}{l}\text { Unfilt. } \\
\text { wshed }\end{array}$ & $\begin{array}{l}\text { Filt } \\
\text { wshed }\end{array}$ \\
\hline Nb of cells & 104 & 100 & 98 & 93 & 86 \\
Total errors & 0 & 6 & 8 & 15 & 18 \\
Over-segmentation errors & 0 & 1 & 2 & 2 & 0 \\
Under-segmentation errors & 0 & 5 & 6 & 13 & 18 \\
\hline
\end{tabular}

On the given image low-resolution image, computing all the 30 LDTs and doing the fits takes 5 seconds on a P-IV $3 \mathrm{GHz}$ PC. On the full data - a $1280 \times 1024$ 12-bit image, the complete computation took about one minute.

\section{Conclusion and Future Work}

In this paper we have presented an algorithm for computing the LDT, the elliptical distance transform and we have provided an illustration of its usefulness. While not exact, it is more accurate than a previously published version, at the cost of increased computational cost. This extra accuracy can be helpful in some applications making use of LDTs.

This algorithm is still open to improvement. In particular an exact version would certainly be desirable. The author is also interested in improving both the precision and the speed of the OUM.

\section{Acknowledgements}

The author wishes to thank Stephen Irrgang for his initial implementation of the LDT. 


\section{References}

1. Rosenfeld, A., Pfaltz, J.: Distance functions on digital pictures. Pattern Recognition 1 (1968) 33-61

2. Borgefors, G.: Distance transformations in arbitrary dimensions. Computer Vision, Graphics, and Image Processing 27 (1984) 321-345

3. Rosenfeld, A., Pfaltz, J.: Sequential operations in digital picture processing. J. Assoc. Comp. Mach. 13(4) (1966) 471-494

4. Calabi, L., Hartnett, W.: Shape recognition prairie fires, convex deficiencies and skeletons. American Mathematical Monthly 75(4) (1968)

5. Borgefors, G.: Distance transformations in digital images. Computer Vision, Graphics, and Image Processing 34 (1986) 344-371

6. Montanari, U.: A method for obtaining skeletons using a quasi-euclidean distance. J. Assoc. Comp. Mach. 15 (1968) 600-624

7. Danielsson, P.E.: Euclidean distance mapping. Computer Graphics and Image Processing 14 (1980) 227-248

8. Vincent, L.: Exact euclidean distance function by chain propagations. In: CVPR, Maui, Hawaii, IEEE (1991) 520-525

9. Cuisenaire, O., Macq, B.: Fast euclidean distance transform by propagation using multiple neighborhoods. Computer Vision and Image Understanding 76(2) (1994) $163-172$

10. Breu, H., Gil, J., Kirkpatrick, D., Werman, M.: Linear time distance transform algorithms. IEEE Transactions on Pattern Analysis and Machine Intelligence 17(5) (1995) 529-533

11. Mehnert, A., Jackway, P.: On computing the exact euclidean distance transform on rectangular and hexagonal grids. Journal of Mathematical Imaging and Vision 11(3) (1999) 223-230

12. Talbot, H., Appleton, B.: Elliptical distance transforms and the object splitting problem. In Talbot, H., Beare, R., eds.: Mathematical Morphology, Proceedings of the 6th international symposium, Sydney, Australia, CSIRO Publishing (2002) $229-240$

13. Rutovitz, D.: Data structures for operations on digital images. In Cheng, G., Ledley, R., Pollok, D., Rosenfel, A., eds.: Pictorial Pattern Recognition, WA, Thomson Book (1968) 105-133

14. Dijkstra, E.: A note on two problems in connexion with graph. Numerische Mathematik 1 (1959) 269-271

15. Beardon, A.: The Geometry of Discrete Groups. Graduate texts in Mathematics. Springer (1983)

16. Sethian, J.: Fast marching methods. SIAM review 41(2) (1999) 199-235

17. Sethian, J.A., Vladimirsky, A.: Ordered upwind methods for static HamiltonJacobi equations. Proc. Nat. Acad. Sciences 98(20) (2002) 11069-11074

18. Serra, J.: Image analysis and mathematical morphology. Academic Press (1982)

19. Soille, P.: Spatial distributions from contour lines: an efficient methodology based on distance transformations. Journal of Visual Communication and Image Representation 2(2) (1991) 138-150

20. Talbot, H., Appleton, B.C.: Elliptical distance transforms and object splitting. In Talbot, H., Beare, R., eds.: Mathematical Morphology: Proceedings of the VIth International Symposium, CSIRO publishing (2002) 229-240

21. Ragnemalm, I.: The euclidean distance transform in arbitrary dimensions. Pattern Recognition Letters 14(11) (1993) 883 - 888 
22. Lantuéjoul, C.: Skeletonization in quantitative metallography. In Haralick, R.M., Simon, J.C., eds.: Issues of Digital Image Procesing, NATO, Sijthoff and Noordhoff (1980)

23. Beucher, S., Vincent, L.: Introduction aux outils morphologiques de segmentation. In: Traitement d'image en microscopie à balayage et en microanalyse par sonde électronique, Paris, ANRT, groupement microanalyse et MEB (1990) F1-F43

24. Talbot, H., Terol Villalobos, I.: Binary image segmentation using weighted skeletons. In: Image algebra and morphological image processing III. Volume 1769., San Diego, CA, SPIE (1992) 147-155

25. Meyer, F.: Cytologie quantitative et morphologie mathématique. PhD thesis, Ecole des Mines de Paris (1979)

26. Talbot, H., Vincent, L.: Euclidean skeleton and conditional bisectors. In: Visual Communications and Image Processing'92. Volume 1818., Boston, SPIE (1992) 862-873

27. Zrour, R., Couprie, M.: Discrete bisector function and euclidean skeleton. In Andres, E., Damiand G., Lienhardt, P., eds.: Discrete Geometry for Computer Imagery: 12th International Conference, DGCI 2005. Volume 3429 of LNCS., Poitiers, France, Springer (2005) 216-227

28. Talbot, H.: Analyse morphologique de fibres minérales d'isolation. PhD thesis, Ecole des Mines de Paris (1993) 